\title{
A Fractional Spline Collocation Method for the Fractional-order Logistic Equation
}

\author{
Francesca Pitolli and Laura Pezza
}

\begin{abstract}
We construct a collocation method based on the fractional B-splines to solve a nonlinear differential problem that involves fractional derivatives, i.e., the fractional-order logistic equation. The use of the fractional B-splines allows us to express the fractional derivatives of the approximating function in an analytical form. Thus, the fractional collocation method is easy to implement, accurate, and efficient. Several numerical tests illustrate the efficiency of the proposed collocation method.
\end{abstract}

Keywords Fractional-order - Logistic equation - Fractional B-spline - Fractional derivative $\cdot$ Collocation method

\section{Introduction}

When we speak about Fractional Derivative, we are generalizing to positive real order the well-understood notion of derivative of integer order (see, for instance, [14]). The nonlocal behavior of the fractional derivative allows to model physical phenomena where memory processes are relevant. For this reason, in the last decades, fractionalorder differential problems were extensively used to describe the anomalous diffusion in porous media, the behavior of viscoelastic materials, the population growth of ecosystems, the intermittent phenomena in plasma, just to cite a few (see, for instance, $[6,9,15]$ and references therein).

On the other hand, the nonlocality is a challenge when one wants to construct numerical methods for the solution of fractional-order differential problems. In fact, to approximate the fractional derivative of a function, its values from the beginning of the discretization interval have to be taken into account. Many numerical methods

F. Pitolli $(\bowtie) \cdot$ L. Pezza

Dept. SBAI, Università di Roma "La Sapienza", Via A. Scarpa 16,

00161 Roma, Italy

e-mail: francesca.pitolli@sbai.uniroma1.it

L. Pezza

e-mail: laura.pezza@sbai.uniroma1.it 
that generalize to fractional-order numerical methods well established in the case of integer-order derivatives, have been proposed in the literature (see, for instance, $[2,3,11,21]$ and references therein). Unfortunately, these methods are computationally demanding when a high accuracy in the numerical solution is required.

In this paper, we construct a collocation method that is especially tailored for fractional-order differential problems. The method looks for an approximating function that belongs to the functional space generated by the fractional B-splines and takes advantage of the explicit formula for their fractional derivatives. The resulting method is easy to implement, accurate, and efficient.

We will use the proposed method to solve a nonlinear fractional differential problem, i.e., the fractional-order logistic differential equation, and we will show that the method produces good approximations while keeping the computational cost low.

The organization of the paper is as follows. In Sect.2, the fractional-order logistic model is presented, and the definition of fractional derivative is given. Section 3 is devoted to the fractional B-splines and their main properties, while the explicit expression of their fractional derivatives is given in Sect. 4. The fractional spline collocation method is introduced in Sect. 5. Finally, in Sect. 6, some numerical tests on the solution of the linear fractional growth model and of the nonlinear fractional logistic model are shown. Some conclusions are drawn in Sect. 7.

\section{The Fractional-order Logistic Differential Equation}

The logistic equation [19]

$$
\left\{\begin{array}{l}
u^{\prime}(t)=\rho u(t)(1-u(t)), \quad t>0, \\
u(0)=u_{0},
\end{array}\right.
$$

where $\rho>0$ is the growth rate, is extensively used to model the growth of a population in the case of limited resources. It has application in several fields, from ecology to chemical reactions, from medicine to transport of goods. Remarkably, the solution of the logistic equation has a closed form, i.e.,

$$
u(t)=\frac{u_{0}}{u_{0}+\left(1-u_{0}\right) \mathrm{e}^{-\rho t}},
$$

also known as the sigmoid function. We notice that Eq. (1) is normalized so that $\lim _{t \rightarrow \infty} u(t)=1$.

Recently, it has been observed that many real-life growth phenomena are better described by fractional-order differential problems (see, for instance, $[1,8,16,17]$ ), so that it seems natural to generalize to the fractional order the logistic model. The fractional-order logistic equation, first analyzed in [4], is 


$$
\left\{\begin{array}{l}
D^{\gamma} u(t)=\rho^{\gamma} u(t)(1-u(t)) \quad t>0, \quad 0<\gamma<1, \\
u(0)=u_{0}
\end{array}\right.
$$

where $D_{t}^{\gamma} u$ denotes the Caputo fractional derivative with respect to the time $t$.

We recall that the Caputo derivative of fractional-order $\gamma>0$ of a function $f \in$ $C^{\lceil\gamma\rceil-1}\left(\mathbb{R}^{+}\right)$having absolute integrable derivative of order $k=\lceil\gamma\rceil$ is defined as

$$
D_{t}^{\gamma} f(t):=\left(\mathscr{J}^{(k-\gamma)} f^{(k)}\right)(t), \quad k-1<\gamma<k, \quad k \in \mathbb{N} \backslash\{0\}, \quad t>0,
$$

where $\mathscr{J}^{(\gamma)}$ is the Riemann-Liouville integral operator

$$
\left(\mathscr{J}^{(\gamma)} f\right)(t):=\frac{1}{\Gamma(\gamma)} \int_{0}^{t} f(\tau)(t-\tau)^{\gamma-1} d \tau
$$

and $\Gamma$ denotes the Euler's gamma function

$$
\Gamma(\gamma):=\int_{0}^{\infty} \tau^{\gamma-1} \mathrm{e}^{-\tau} d \tau
$$

Here, $\mathbb{N}$ denotes the set of nonnegative integers.

For details on fractional calculus and fractional derivatives see, for instance, [14].

The existence and uniqueness of the solution to (3) was proved in [4], while its analytical solution was given in [20],

$$
u(t)=\sum_{k=0}^{\infty}\left(\frac{u_{0}-1}{u_{0}}\right)^{k} E_{\gamma}\left(-k \rho^{\gamma} t^{\gamma}\right)
$$

where $E_{\gamma}(z)$ is the Mittag-Leffler function defined as

$$
E_{\gamma}(z)=\sum_{k=0}^{\infty} \frac{z^{k}}{\Gamma(k \gamma+1)}
$$

The fractional-order influences the population growth: The lower the derivative order is, the slower the rate of growth becomes.

In the following, we will construct a collocation method based on the fractional B-splines to solve the fractional logistic equation.

\section{The Fractional B-Splines}

The fractional B-splines, i.e., the B-splines of fractional order, were introduced in [18] generalizing to fractional power the classical definition of polynomial B-splines of integer order. We notice that here 'fractional' actually means 'real.' In fact, let 


$$
t_{+}^{\alpha}:=\left\{\begin{array}{ll}
t^{\alpha}, & t \geq 0, \\
0, & \text { otherwise },
\end{array} \quad \alpha>-1 / 2\right.
$$

be the fractional truncated power function, and let $\Delta_{h}^{\gamma}$ be the generalized finite difference operator

$$
\Delta_{h}^{\gamma} f(t):=\frac{1}{h^{\gamma}} \sum_{k=0}^{\infty}(-1)^{k}\left(\begin{array}{l}
\gamma \\
k
\end{array}\right) f(t-h k), \quad \gamma \in \mathbb{R}^{+},
$$

where

$$
\left(\begin{array}{l}
\gamma \\
k
\end{array}\right):=\frac{\Gamma(\gamma+1)}{k ! \Gamma(\gamma-k+1)}, \quad k \in \mathbb{N}, \quad \gamma \in \mathbb{R}^{+},
$$

are the generalized binomial coefficients. When $\gamma \in \mathbb{N} \backslash\{0\},\left\{\left(\begin{array}{l}\gamma \\ k\end{array}\right)\right\}$ is the usual binomial coefficient sequence so that the series in (10) is a finite sum and $\Delta_{h}^{\gamma}$ reduces to the usual finite difference operator. When $\gamma \in \mathbb{R}^{+} \backslash \mathbb{N}$, the sequence $\left\{\left(\begin{array}{l}\gamma \\ k\end{array}\right)\right\}$ is no more compactly supported even if it is absolutely summable so that the limit of the series (10) exists under suitable conditions on $f$.

The fractional $B$-spline $B_{\alpha}$ of order $\alpha$ is defined as

$$
B_{\alpha}(t):=\frac{\Delta_{h}^{\alpha+1} t_{+}^{\alpha}}{\Gamma(\alpha+1)}, \quad \alpha>-\frac{1}{2} .
$$

When $\alpha=n \in \mathbb{N}, B_{n}$ is the well-known polynomial B-spline of integer degree $n$ and compact support [0,n+1]. When $\alpha$ is not an integer, the fractional B-spline $B_{\alpha}$ does not have compact support but it decays as

$$
B_{\alpha}(t) \rightarrow \frac{1}{|t|^{-\alpha-2}}
$$

for $t \rightarrow \infty$ and belongs to $L^{2}(\mathbb{R})$.

The fractional B-splines for different values of the parameter $\alpha$ are displayed in Fig. 1 (top left panel). The classical polynomial B-splines are also displayed (dashed lines). The picture shows that even if the fractional B-splines do not have compact support, they decay very fast so that they can be truncated for computational purposes. Moreover, we observe that the fractional B-splines are not always positive, even if the nonnegative part becomes smaller and smaller when $\alpha$ increases. 

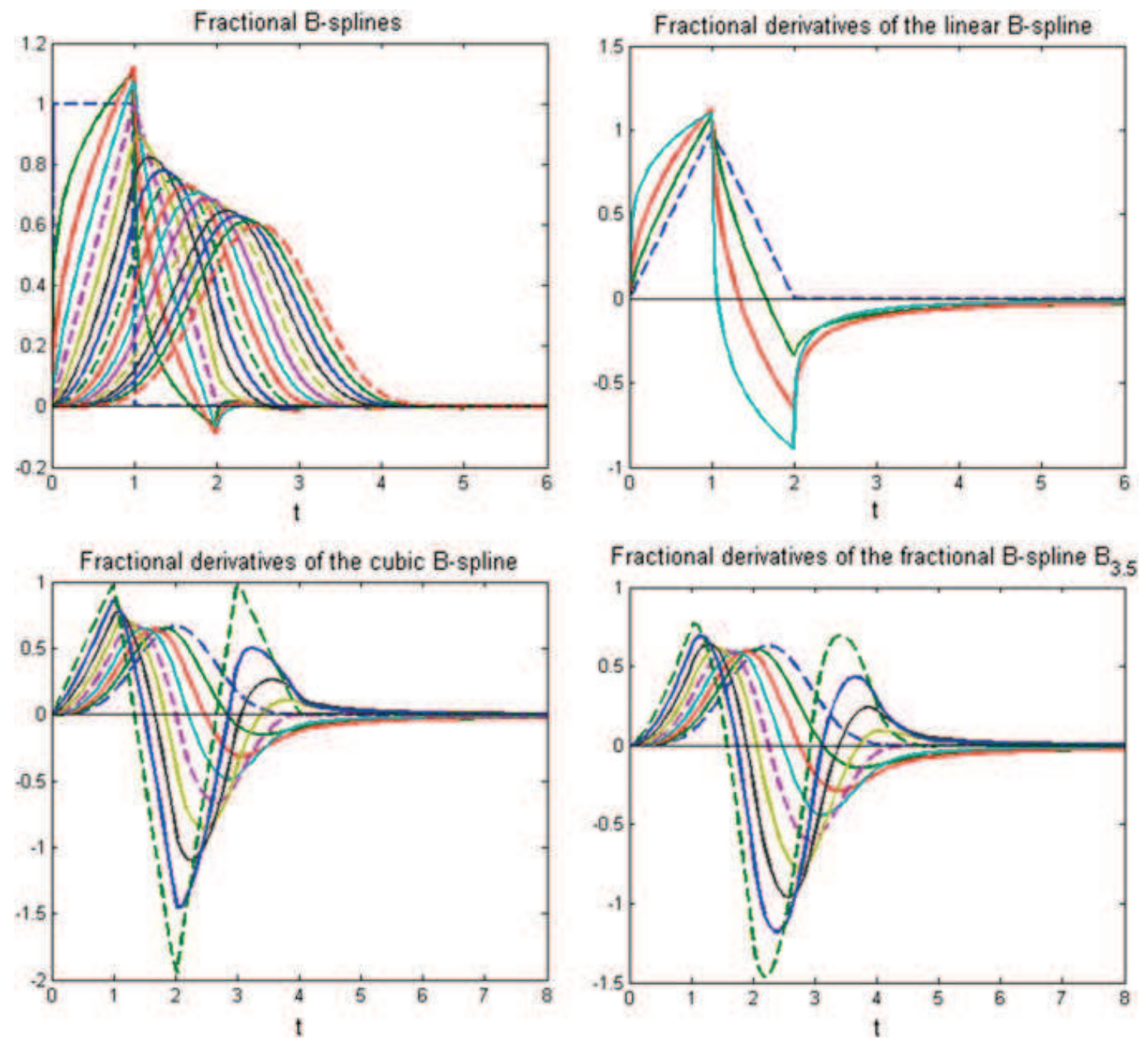

Fig. 1 The fractional B-splines (solid lines) and the polynomial B-splines (dashed lines) for $\alpha$ ranging from 0 to 4 (top left panel). The fractional derivatives of the linear B-spline $B_{1}$ for $\gamma=$ $0.25,0.5,0.75$ (top right panel). The fractional derivatives of the cubic B-spline $B_{3}$ (bottom left panel) and of the fractional B-spline $B_{3.5}$ (bottom right panel) for $\gamma$ ranging from 0.25 to 2 . Ordinary derivatives are displayed as dashed lines

\section{The Fractional Derivatives of the Fractional B-Splines}

Fractional derivatives of fractional B-splines can be evaluated by differentiating (12). First of all we observe that the fractional derivative of order $\gamma$ of the fractional truncated power (9) can be evaluated explicitly using definition (4). We get

$$
D_{t}^{\gamma} t_{+}^{\alpha}=\left\{\begin{array}{lll}
\frac{\Gamma(\alpha+1)}{\Gamma(\alpha+1-\gamma)} t_{+}^{\alpha-\gamma}, & t>0, & 0<\gamma \leq \alpha . \\
0, & \text { otherwise, }
\end{array}\right.
$$


Substituting (14) in (12), we get the differentiation rule

$$
D_{t}^{\gamma} B_{\alpha}(t)=\frac{\Delta_{h}^{\alpha+1} t_{+}^{\alpha-\gamma}}{\Gamma(\alpha-\gamma+1)}, \quad 0<\gamma<\alpha+\frac{1}{2},
$$

which reduces to the differentiation rule for the classical B-splines when $\gamma, \alpha \in \mathbb{N}$.

We observe that since $B_{\alpha}$ is a causal function with $B_{\alpha}^{(n)}(0)=0$ for $n \in \mathbb{N} \backslash\{0\}$, the Caputo fractional derivative coincides with the Riemann-Liouville fractional derivative defined as

$$
D_{t}^{\gamma} f(t):=\frac{d^{k}}{d t^{k}}\left(\mathscr{J}^{(\gamma)} f\right)(t), \quad k-1<\gamma<k, \quad k \in \mathbb{N}, \quad t>0 .
$$

It is interesting to note that the fractional derivative of a fractional B-spline of degree $\alpha$ can be expressed as a linear combination of translates of a fractional B-spline of lower degree, i.e., $D_{t}^{\gamma} B_{\alpha}$ is a fractional spline. Since the differentiation rule (15) holds also when $\alpha$ is an integer, the fractional derivatives of the classical polynomial B-splines are fractional splines, too. As a consequence, $D_{t}^{\gamma} B_{n}$ is not compactly supported when $\gamma \in \mathbb{R}^{+} \backslash \mathbb{N}$ reflecting the nonlocal behavior of the fractional derivatives. In Fig. 1, the fractional derivatives of $B_{1}$ (top right panel), $B_{3}$ (bottom left panel), and $B_{3.5}$ (bottom right panel) are displayed for different values of $\gamma$.

\section{The Fractional Spline Collocation Method}

The fractional spline collocation method looks for an approximating function that belongs to the space of the fractional spline $B_{\alpha}$. Let $\Delta=\{j h, 0 \leq j \leq N\}, N=$ $T / h$, be a partition of equispaced nodes in the interval $[0, T]$ and let $\mathscr{V}_{\alpha, h}$ be the fractional spline space on the partition $\Delta$. As a basis for $\mathscr{V}_{\alpha, h}$, we choose the set of functions

$$
\Phi_{\alpha, h, k}=\left\{\varphi_{\alpha, h, k}(t)=B_{\alpha}(t-h k), k \in \mathscr{N}_{h}, t \in[0, T]\right\}
$$

Here, $\mathscr{N}_{h}$ is a suitable set of indexes chosen in order to take into account all the translates of $B_{\alpha}$ that are significantly different from zero in [0,T]. When $\alpha=n \in \mathbb{N}$, due to the compact support of $B_{n}$ we get $\mathscr{N}_{h}=\{k,-n \leq k \leq N\}$. When $\alpha$ is not an integer, since $B_{\alpha}$ decays fast toward infinity, for computational purposes we can assume supp $B_{\alpha} \approx\left[0, N_{0}\right]$, where $N_{0} \geq\lceil\alpha\rceil+2$ is a truncation parameter (cf. Fig. 1). Thus, $\mathscr{N}_{h}=\left\{k,-N_{0}+1 \leq k \leq N\right\}$. We observe that the basis $\Phi_{\alpha, h, k}$ has a certain number of boundary functions, depending on the value of $\alpha$. More precisely, let $N_{\alpha}$ be an integer equal to $n$ when $\alpha$ has an integer value and equal to $N_{0}$ when $\alpha$ is not an integer. Then, the basis $\Phi_{\alpha, h, k}$ has $2 N_{\alpha}$ boundary functions, i.e., the $N_{\alpha}$ functions $\varphi_{\alpha, h, k}(t)$ with index $k$ ranging from $-N_{\alpha}$ to -1 (left boundary), and the $N_{\alpha}$ functions 
$\varphi_{\alpha, h, k}(t)$ with index $k$ ranging from $N-N_{\alpha}+1$ to $N$ (right boundary). Here, the boundary functions are obtained just restricting $B_{\alpha}(t-h k)$ to the interval $[0, T]$. i.e.,

For a given partition $\Delta$, we look for an approximating function $u_{\alpha, h}(t) \in \mathscr{V}_{\alpha, h}$,

$$
u_{\alpha, h}(t)=\sum_{k \in \mathscr{N}_{h}} \lambda_{\alpha, h, k} \varphi_{\alpha, h, k}(t)
$$

which solves the differential problem (3) on a set of collocation points. Here, we choose as collocation points the set of equispaced nodes $\left\{t_{p}=p \delta, p=0, \ldots, M\right\}$, where $M=T / \delta$. Thus, the discretized version of (3) reads

$$
\left\{\begin{array}{l}
D_{t}^{\gamma} u_{\alpha, h}\left(t_{p}\right)=\rho^{\gamma} u_{\alpha, h}\left(t_{p}\right)\left(1-u_{\alpha, h}\left(t_{p}\right)\right), \quad p=1, \ldots, M \\
u_{\alpha, h}(0)=u_{0}
\end{array}\right.
$$

Substituting (18) in (19), we get

$$
\left\{\begin{array}{l}
\sum_{k \in \mathscr{N}_{h}} \lambda_{k} D_{t}^{\gamma} \varphi_{k}\left(t_{p}\right)=\rho^{\gamma} \sum_{k \in \mathscr{N}_{h}} \lambda_{k} \varphi_{k}\left(t_{p}\right)\left(1-\sum_{k \in \mathscr{N}_{h}} \lambda_{k} \varphi_{k}\left(t_{p}\right)\right), \quad p=1, \ldots, M, \\
\sum_{k \in \mathscr{N}_{h}} \lambda_{k} \varphi_{k}(0)=u_{0},
\end{array}\right.
$$

where, for short notation, we dropped the subscripts $\alpha$ and $h$.

Equations (20) form a nonlinear system of $M$ equations for the $N \leq M$ unknowns $\left\{\lambda_{k}, k \in \mathscr{N}_{h}\right\}$ and can be solved, for instance, by the Levenberg-Marquardt algorithm. In the following section, we give some numerical results showing the feasibility of the method.

\section{Numerical Tests}

In this section, we will show the performance of the proposed collocation method in solving some test problems.

First of all, we test the fractional spline collocation method on the fractional growth equation

$$
\left\{\begin{array}{l}
D^{\gamma} u(t)=\rho^{\gamma} u(t), \quad t>0, \quad 0<\gamma \leq 1, \quad \rho>0, \\
u(0)=u_{0}
\end{array}\right.
$$


which generalizes to the fractional-order derivative the well-known Malthusian model [10]. Its analytical solution, obtained through the Laplace transform, has the following expression (cf. [20])

$$
u(t)=u_{0} E_{\gamma}\left((\rho t)^{\gamma}\right) .
$$

It reduces to the classical exponential growth law when $\gamma=1$.

Applying to Eq. (21) the collocation method highlighted in the previous section, we get the linear system

$$
\left\{\begin{array}{l}
\sum_{k \in \mathscr{N}_{h}} \lambda_{k} D_{t}^{\gamma} \varphi_{k}\left(t_{p}\right)-\rho^{\gamma} \sum_{k \in \mathscr{N}_{h}} \lambda_{k} \varphi_{k}\left(t_{p}\right)=0, \quad p=1, \ldots, M, \\
\sum_{k \in \mathscr{N}_{h}} \lambda_{k} \varphi_{k}(0)=u_{0},
\end{array}\right.
$$
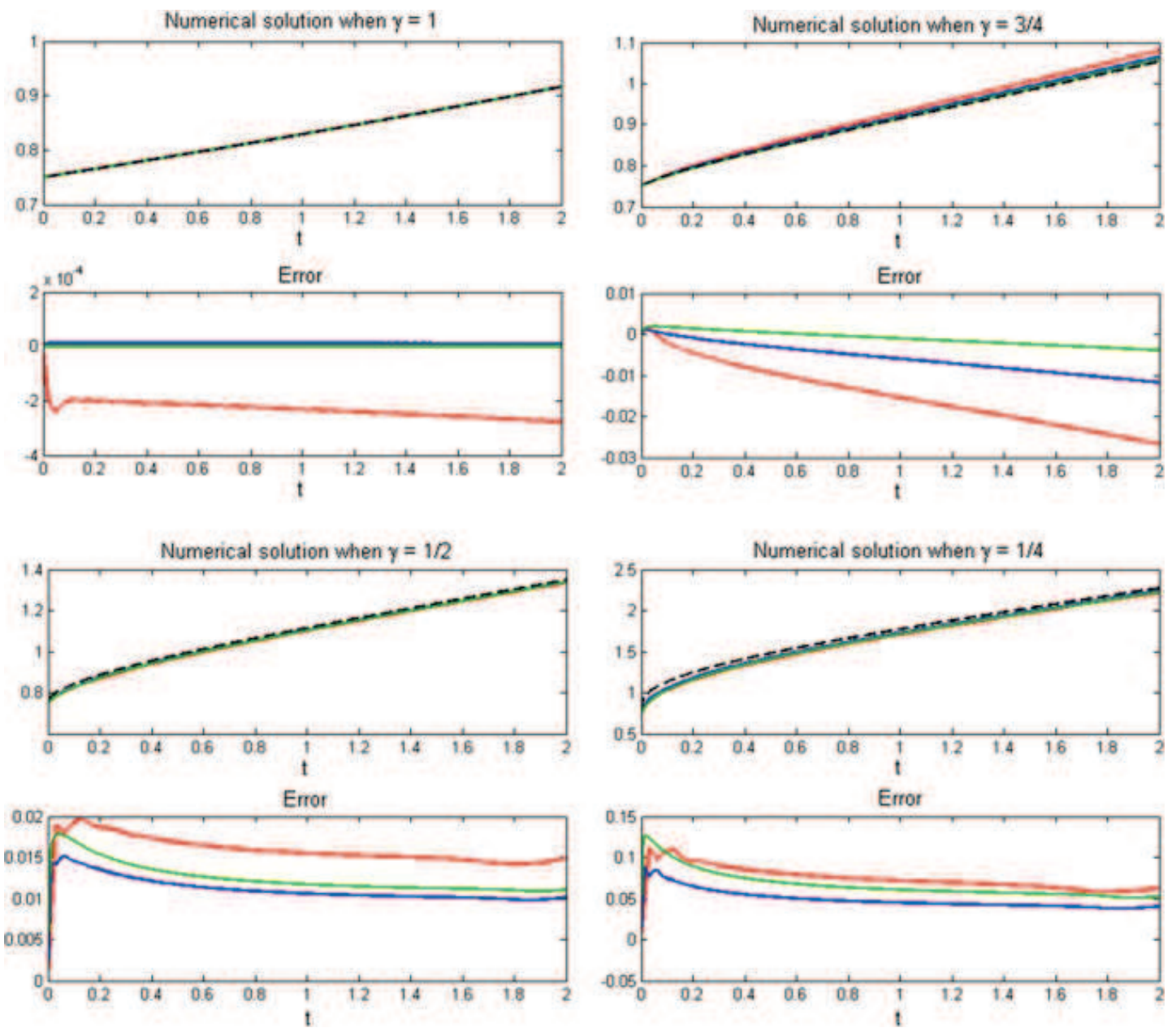

Fig. 2 Fractional growth model: The numerical solution and the error obtained by using the fractional B-spline $B_{3.75}$ for $\gamma=1$ (top left panels), 3/4 (top right panels), 1/2 (bottom left panels), $1 / 4$ (bottom right panels) for increasing values of $M$ and $N$; i.e., $M=64, N=37$ (red); $M=128$, $N=69$ (blue); $M=256, N=133$ (green). The analytical solution is shown as a dashed black line 
where $\left\{\lambda_{k}\right\}$ are the $N$ unknowns. When $N<M$, the linear system is overdetermined and can be solved in the least squares sense.

In the following numerical tests, we set $\rho=1$.

The numerical solution and the error $u(t)-u_{\alpha, h}(t)$ obtained when $\alpha=3.75$ are shown in Fig. 2 for different values of $\gamma$ and decreasing values of the partition step $h$ and of the collocation step $\delta$, i.e., $\gamma=1,3 / 4,1 / 2,1 / 4, h=2^{-4}, 2^{-5}, 2^{-6}$, and $\delta=2^{-5}, 2^{-6}, 2^{-7}$. Here, the truncation parameter $N_{0}$ is set equal to 6 so that $N=$ $T / h+N_{0}-1=37,69,133$ while $M=T / \delta=64,128,256$.

Figure 3 displays the numerical solution and the error obtained by using the fractional B-splines $B_{\alpha}$ with $\alpha=3,3.25,3.5,3.75,4$, for the two values $\gamma=1 / 4$ and $\gamma=1$ (Malthus model).

These simple tests show that the numerical solution obtained by the proposed collocation method has a good accuracy even in the case of few nodes and low dimension of the approximating space. Moreover, a suitable choice of the fractional order of the fractional B-spline can improve the approximation accuracy.

Then, we solved the fractional logistic equation using the same parameters as in the previous tests. The numerical solution and the error obtained when $\alpha=3.75$ are shown in Fig. 4 for different values of $\gamma$. Figure 5 displays the numerical solution and the error obtained by using the fractional B-splines $B_{\alpha}$ with $\alpha=3,3.25,3.5$, $3.75,4$, for the two cases $\gamma=1 / 4$ and $\gamma=1$.

The numerical results show that also for this nonlinear problem, the collocation method provides an accurate solution.
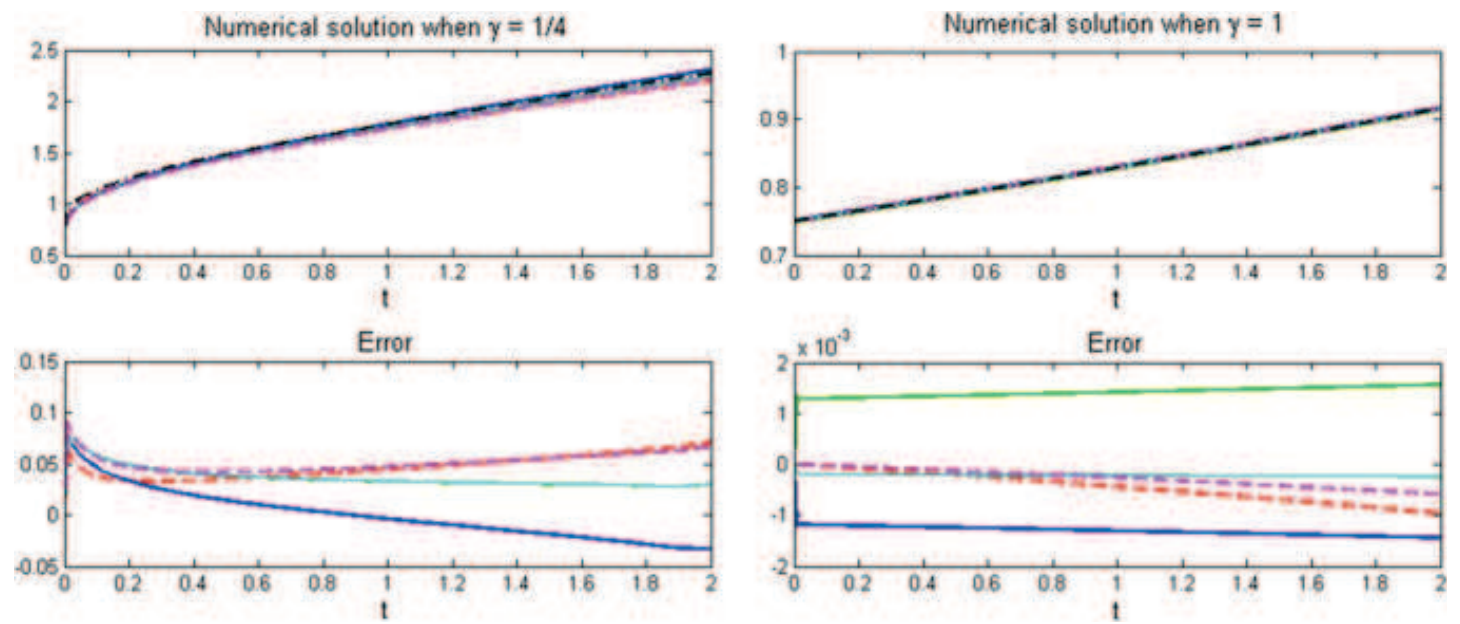

Fig. 3 Fractional growth model: The numerical solution and the error for $\gamma=1 / 4$ (left), $\gamma=1$ (right) obtained by using the fractional B-splines $B_{3.25}$ (blue), $B_{3.5}$ (green), $B_{3.75}$ (cyan). Dashed lines correspond to the polynomial B-splines $B_{3}$ (red) and $B_{4}$ (magenta). Here, $M=128, N=69$ 

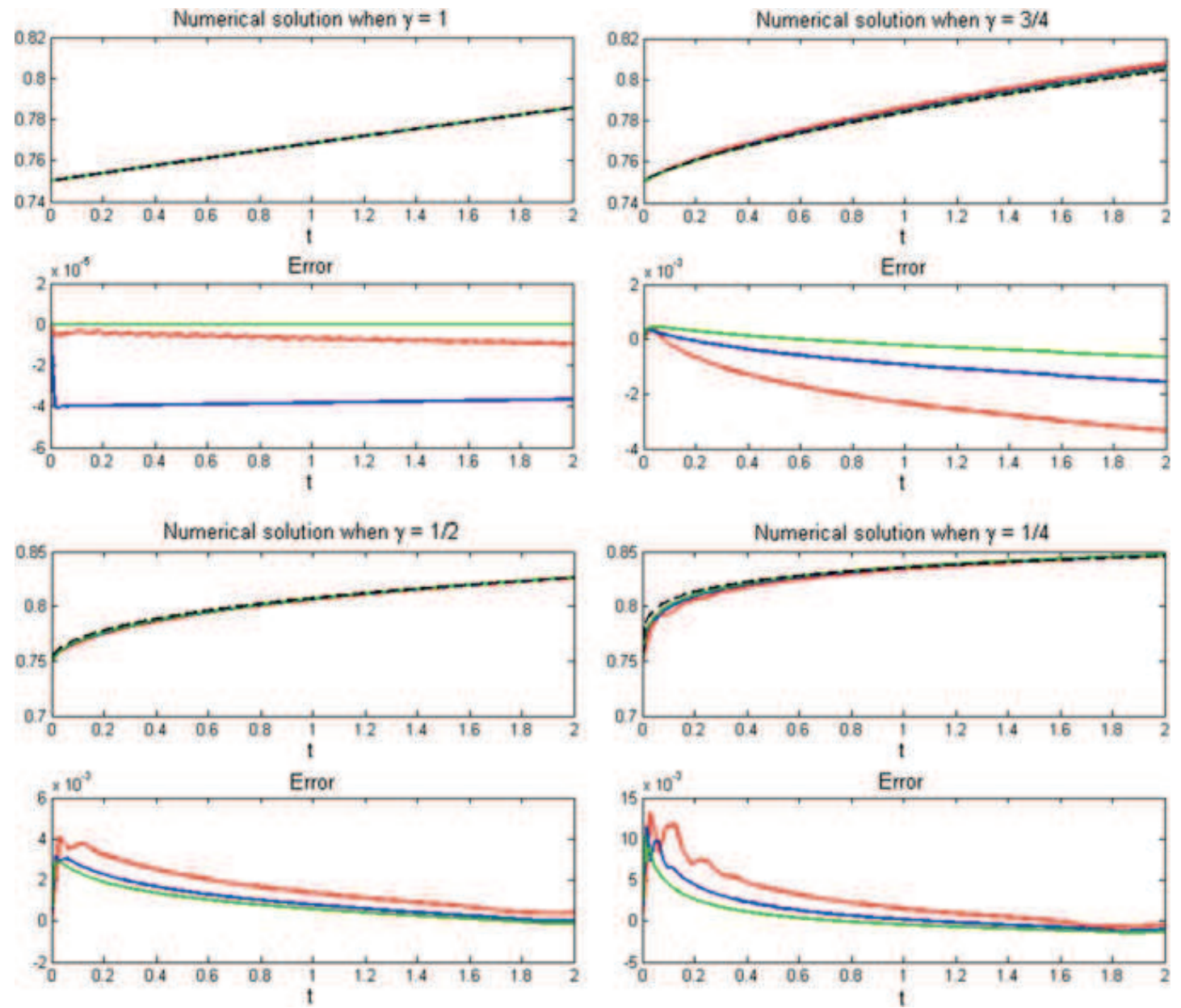

Fig. 4 Fractional logistic model: The numerical solution and the error obtained by using the fractional B-spline $B_{3.75}$ for $\gamma=1$ (top left panels), 3/4 (top right panels), 1/2 (bottom left panels), $1 / 4$ (bottom right panels) for increasing values of $M$ and $N$, i.e., $M=64, N=37$ (red); $M=128$, $N=69$ (blue); $M=256, N=133$ (green). The analytical solution is shown as a dashed black line
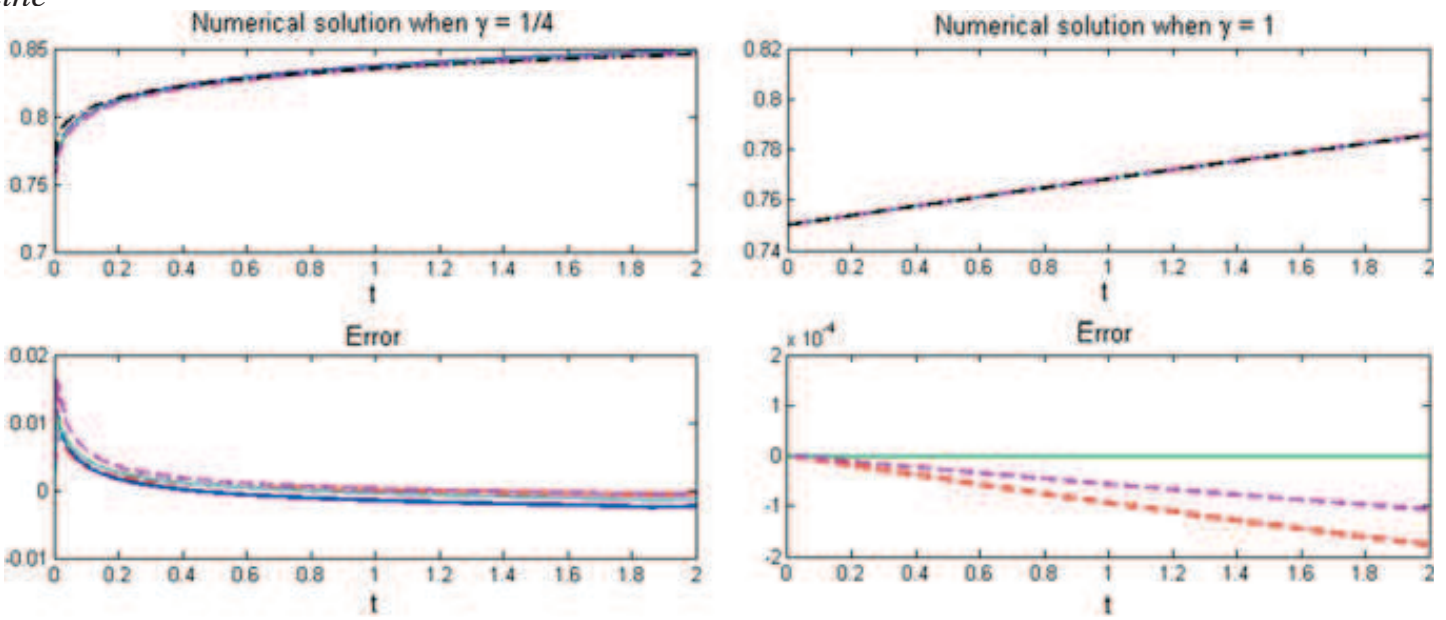

Fig. 5 Fractional logistic model: The numerical solution and the error for $\gamma=1 / 4$ (left panels), $\gamma=1$ (right panels) obtained by using the fractional B-splines $B_{3.25}$ (blue), $B_{3.5}$ (green), $B_{3.75}$ (cyan). Dashed lines corresponds to the polynomial B-splines $B_{3}($ red $)$ and $B_{4}$ (magenta). Here, $M=128, N=69$ 


\section{Conclusion}

We presented a fractional spline collocation method suitable to solve both linear and nonlinear fractional-order differential problems, and we tested their performance on the growth and logistic differential equations of fractional order. The numerical results show that the proposed method produces an accurate approximation with a low computational cost. Moreover, the order of the fractional B-spline to be used in the method can be considered as a free parameter and can be chosen in order to reduce the approximation error.

A theoretical proof of the convergence when $h$ and $\delta$ go to zero can be given following classical arguments in approximation theory (cf. [13]) and will be the subject of a forthcoming paper where the use of different approximating operators and different node distributions will be also considered. Moreover, the additional approximation error introduced by the truncation parameter $N_{0}$ has to be carefully evaluated.

Another issue to be taken into account is the truncation of the boundary functions since it could generate numerical instabilities due to the possibly high condition number of the corresponding collocation matrix. There exist different approaches to obtain stable bases on finite intervals. Since the fractional B-splines are refinable functions and generate a multiresolution analysis in $L_{2}(\mathbb{R})$, we can adapt to the fractional B-spline case some methods already used for constructing refinable bases on the interval (see, for instance, [5]).

As a final remark, we observe that refinability is a key ingredient to construct fractional wavelet collocation methods (cf. [12]).

\section{References}

1. R. Almeida, N.R.O. Bastos, M.T.T. Monteiro, Modeling some real phenomena by fractional differential equations. Math. Meth. Appl. Sci. 39, 4846-4855 (2016)

2. D. Baleanu, K. Diethelm, E. Scalas, J.J. Trujillo, Fractional Calculus, Models and Numerical Methods (World Scientific, Singapore, 2012)

3. S. Das, P.K. Gupta, Approximate approach to the Das model of fractional logistic population growth. Appl. App. Math. 5, 605-611 (2010)

4. A.M.A. El-Sayed, A.E.M. El-Mesiry, H.A.A. El-Saka, On the fractional-order logistic equation. Appl. Math. Lett. 20, 817-823 (2007)

5. L. Gori, L. Pezza, F. Pitolli, Recent results on wavelet bases on the interval generated by GP refinable functions. Appl. Num. Math. 51, 549-563 (2004)

6. R. Hilfer (ed.), Applications of Fractional Calculus in Physics (World Scientific Publishing Co., Inc, River Edge, NJ, 2000)

7. A.A. Kilbas, H.M. Srivastava, J.J. Trujillo, Theory and Applications of Fractional Differential Equations. North-Holland Mathematics Studies, vol. 204 (Elsevier Science, Amsterdam, 2006)

8. Y.F. Luchko, M. Rivero, J.J. Trujillo, M.P. Velasco, Fractional models, non-locality, and complex systems. Comput. Math. App. 59, 1048-1056 (2010)

9. F. Mainardi, Fractional Calculus and Waves in Linear Viscoelasticity: An Introduction to Mathematical Models (Imperial College Press, London, 2010) 
10. T.R. Malthus, An Essay on the Principle of Population (1798) (Macmillan \& Co., London, 1926)

11. A. Pedas, E. Tamme, Numerical solution of nonlinear fractional differential equations by spline collocation methods. J. Comput. Appl. Math. 255, 216-230 (2014)

12. L. Pezza, F. Pitolli, A fractional wavelet Galerkin method for the fractional diffusion problem. IMACS Ser. Comput. Appl. Math. 20, 1-10 (2017)

13. L. Pezza, F. Pitolli, A multiscale collocation method for fractional differential problems. Math. Comput. Simul

14. I. Podlubny, Fractional Differential Equations. Mathematics in Science and Engineering, vol. 198 (Academic Press, Cambridge, 1999)

15. V.E. Tarasov, Fractional Dynamics. Applications of Fractional Calculus to Dynamics of Particles, Fields and Media. Nonlinear Physical Science (Springer, Heidelberg, 2010)

16. V.V. Tarasova, V.E. Tarasov, Logistic map with memory from economic model. Chaos, Solitons Fractals 95, 84-91 (2017)

17. R. Toledo-Hernandez, V. Rico-Ramirez, G.A. Iglesias-Silva, U.M. Diwekar, A fractional calculus approach to the dynamic optimization of biological reactive systems. Part I: Fractional models for biological reactions. Chem. Eng. Sci. 117, 217-228 (2014)

18. M. Unser, T. Blu, Fractional splines and wavelets. SIAM Rev. 42, 43-67 (2000)

19. P.F. Verhulst, Notice sur la loi que la population suit dans son accroissement. Correspondance mathématique et physique, Quetelet 10, 113-121 (1838)

20. B.J. West, Exact solution to fractional logistic equation. Physica A 429, 103-108 (2015)

21. M.Zayernouri, G.E. Karniadakis, Fractional spectral collocation method. SIAM J. Sci. Comput. 36, A40-A62 (2014) 